\section{Conquering the barriers: are antibody therapeutics feasible for CNS indications?}
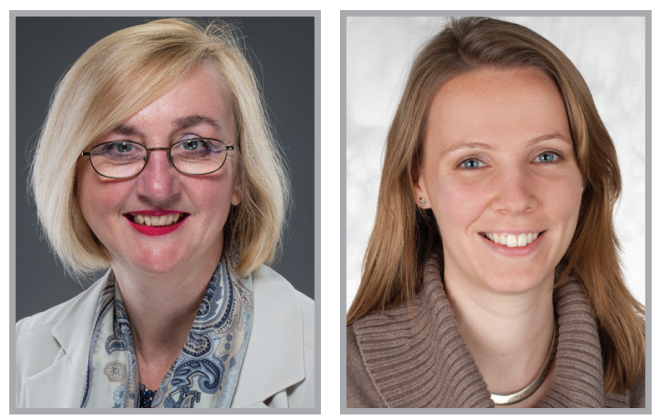

\author{
“...the future for CNS antibody \\ therapeutics has just become \\ much brighter."
}

\begin{abstract}
Lagging behind: barriers to CNS drug development

The major hurdles on the development path of CNS therapeutics include an inherent complexity of the CNS, highly restricted drug access to CNS targets by the blood-brain barrier (BBB), paucity of translational preclinical disease models, safety risks, and lack of clinical biomarkers to aid in patient selection and early assessment of efficacy in clinical trials. Drug development for CNS is therefore highly complex and risky - more expensive and lengthy than for any other indication. The CNS drugs have one of the highest pipeline attrition rates and longest development times (114 months); clinical trial failures tend to occur later in the clinical development, making the cost of developing a CNS drug among the highest of any therapeutic indication $[1,2]$.
\end{abstract}

Antibody drugs have become an increasingly significant component of the therapeutic landscape, achieving higher approval rates compared with nonbiologics (synthetic molecules). Antibodies exhibit very high specificity and selectivity, reducing risks of 'off-target' toxicity typical for synthetic molecules and enabling 'personalized' treatments [3]. Driven by clinical and commercial successes of 'the first wave' of therapeutic antibodies (e.g., anti-HER2 Trastuzumab in breast cancer and antiTNF $\alpha$ Humira in rheumatoid arthritis), the antibody pipelines for oncology and inflammatory diseases have swelled to over 130 candidates in Phase 1 or beyond [1-3]. Regrettably, CNS indications have been left behind in this biologics 'revolution,' missing sorely the opportunity to benefit from novel, precise, disease-modifying antibody therapeutics. The overwhelming majority of all CNS therapeutics remain small molecules and there are currently no approved therapeutic antibodies for nonimmunological neurological diseases.

The cause for this 'state of affairs' has not been lack of trying. For example, immunotherapy for Alzheimer's disease (AD) has generated intense interest and led to the development of therapeutic antibodies against amyloid $\beta(A \beta)$ and tau proteins [4]. Antibodies generated against various epitopes of $A \beta$ proceeded to human trials based on the belief that the elimination of brain amyloid deposits would be

\section{KEYWORDS}

- bispecific antibodies • blood-brain barrier • CNS • drug development

"The CNS drugs have one of the highest pipeline attrition rates and longest development times

(114 months); clinical trial failures tend to occur later in the clinical development, making the cost of developing a CNS drug among the

highest of any therapeutic indication." 
"...for the first time since the development of the molecular blood-brain barrier Trojan concept, the field has at its disposal a panel of blood-brain

barrier 'carriers' built into

blood-brain barriercrossing bispecific proteins validated in preclinical models, some of which are on their path to clinical trials." accelerated by 'mopping up' and removing circulating amyloid - without much consideration given as to whether and which levels of antibodies are required in the brain parenchyma to 'engage' and remove central A $\beta$ deposits. Among active and passive anti-A $\beta$ immunotherapies for $\mathrm{AD}$, bapineuzumab and solanezumab, two humanized monoclonal antibodies, failed to show significant clinical benefits in mild-tomoderate AD patients in large Phase III clinical trials [5]. It is now believed that predominantly peripherally restricted actions of $A \beta$ antibodies are not sufficient and that their delivery across the BBB is required to produce a central therapeutic effect. Indeed, $A \beta$-binding antibody fragments delivered centrally were highly effective in preventing $A \beta$-deposition in transgenic models [6]. These expensive clinical failures of AD immunotherapy were instrumental in recognizing the need to integrate the development of BBB-delivery technologies into preclinical and clinical development of biotherapeutics for CNS indications.

\section{BBB Trojans: a path forward for CNS therapeutic antibodies?}

A highly restricted delivery of therapeutic antibodies across the BBB, usually estimated at less than $0.1 \%$ of injected dose, is compounded by their very limited diffusion within the brain parenchyma and the general lack of knowledge and experience regarding their (on target) safety liabilities within the CNS. The elimination pathways of antibodies from the CNS are poorly understood and their species selectivity often creates difficulties in translating findings from preclinical models. Faced with these barriers, one might question whether antibody therapeutics are feasible for CNS targets hidden beyond brain barriers?

Auspiciously, the hope for CNS therapeutic antibodies has been kept alive by recent evidence of highly improved preclinical central efficacy achieved by linking a therapeutic antibody with an antibody that undergoes transport across the $\mathrm{BBB}$.

These BBB-crossing bispecific antibodies exploit a molecular Trojan horse concept described in the early 1990s [7]. Certain BBB receptors, including transferrin receptor (TfR), insulin receptor and LRP-1, deliver their ligands into the brain by constant shuttling between luminal and abluminal (brain-facing) membranes of brain endothelial cells, a process known as receptor-mediated transcytosis (RMT) [8]. Antibodies or peptides that bind these receptors have been engineered as molecular Trojan horses to deliver genetically or chemically linked therapeutic payloads via these physiological 'shuttles.' For example, a humanized mouse monoclonal antibody against human insulin receptor (HIRMAb) has been used as BBB-delivery Trojan with various payloads genetically fused onto its C-terminus to create bispecific tetrameric molecules. This HIRMAb-enzyme idunorate 2 -sulfatase fusion [9] has recently proceeded into clinical trials for the orphan lysosomal storage disease, mucopolysaccharoidosis Type II (Hunter's syndrome).

Antibodies against the TfR, described as first brain-delivery Trojans [7], have inspired a renewed interest after Genentech demonstrated improved brain delivery of a bispecific heterodimeric antibody combining a low-affinity binding anti-TfR arm and a high-affinity therapeutic arm against the amyloid processing enzyme BACE1 [10]. This antibody has since undergone further engineering to derisk immune-mediated 'on-target' side effects [11] and to produce variants that cross-react with human and nonhuman primate TfR receptors [12]. Intravenous dosing of monkeys with anti-TfR/BACE1 antibodies reduced $A \beta$ both in cerebral spinal fluid and in brain tissue and the degree of reduction correlated with the brain concentration of anti-TfR/BACE1 antibody [12]. After this compelling demonstration of the pharmacokinetic-pharmacodynamic (PK-PD) relationship of the anti-TfR/BACE1 bispecific antibody, several biotechnology companies followed suite with different variants of TfR antibodies, including among others, a monovalent TfR Fab fused to the C-terminus of an anti-A $\beta$ antibody [13], a dual variable domain IgGs [14] and a mouse-human cross-reactive individual variable domains of shark heavy chain-only antibodies (known as VNARs) [15]. Collectively, these developments contributed to an improved understanding of the mechanisms governing TfR-mediated RMT and, implicitly, of antibody engineering and design requirements to increase their $\mathrm{BBB}$ transcytosis efficiency. Optimization of the RMT shuttle-antibody binding affinity [10-12] and dissociation constants, especially at acidic $\mathrm{pH}$ typical of endosomal compartment(s), adjustment of valency of antibody-receptor binding to mitigate receptor cross-linking and degradation [13], selection of binding epitopes which do not interfere with natural ligand binding and transport, and 
mitigation of 'on-target' toxicity through removal of effector function [11] emerged from these studies as 'guiding principles' for engineering BBB-crossing bispecific antibodies.

In parallel, a pursuit of new BBB 'shuttle' receptors with better BBB specificity - to avoid the peripheral 'sink' observed with the widely distributed TfR - and with increased RMT potential led to the discovery of new BBB Trojan antibodies through application of genomic, proteomic and phenotypic screening approaches [16]. One such BBB Trojan, the single-domain antibody FC5, was identified by phenotypic panning of a camelid phage-display library to identify candidates that undergo binding and internalization into human brain endothelial cells and can transcytose across an in vitro BBB model [17]. This 'small' $(15 \mathrm{kD})$ antibody fragment boasts an exceptional 'modularity' for designing various $\mathrm{BBB}$-crossing bispecific molecules due to its compact and monomeric features [18]. Incorporated as a building block in various antibody-like molecules, it enhanced their cross$\mathrm{BBB}$ transport in vitro and in vivo in either monoor bivalent formats [19]. Antibodies engineered with FC5 as BBB-crossing 'arm' showed up to a 20 -fold increase in cerebral spinal fluid exposure compared with control antibodies and elicited dose-dependent pharmacological responses on central targets [19]. These studies demonstrated that rationally designed phenotypic selection approaches have the potential to yield novel BBB-crossing Trojan antibodies with desired characteristics, platform properties and improved developability.

With re-engineered 'old' and emerging 'new' BBB molecular Trojans, the bispecific antibody therapeutics for CNS have entered a realm of feasible. However, many hurdles remain. The currently used Trojans all have drawbacks, including wide tissue distribution of their targets and potential safety liabilities (for review, see [18]). The greatest looming risk for the overall concept is the demonstration of translation into humans.

To facilitate this translation to clinical proof of concept, the field will have to bridge the current gap in understanding not only the antibody transport across the BBB, but also their 'fate' in the brain proper, including diffusion to target, target-mediated disposition and degradation, elimination via brain 'glymphatic' circulation [20] and active transport. This knowledge in combination with PK-PD studies with BBB-crossing bispecific antibodies will provide the basis for building more accurate and scalable PK-PD models which incorporate surrogate biomarkers of target engagement and efficacy.

In summary, for the first time since the development of the molecular BBB Trojan concept [9], the field has at its disposal a panel of BBB 'carriers' built into $\mathrm{BBB}$-crossing bispecific proteins validated in preclinical models, some of which are on their path to clinical trials. These and future BBB-delivery technologies with improved selectivity, efficacy and safety profiles and broad applicability with different classes of therapeutics, including biologics, could become a 'game changer' in expanding biotherapeutic applications to CNS diseases. In addition to accelerating the development of therapeutic antibodies for CNS indications, these technologies will become instrumental in validating a myriad of potential old and new CNS targets, previously hidden beyond brain barriers, using more precise targeting and modulation afforded by antibodies. The emerging pipeline of BBB molecular Trojans and promising results from preclinical development of BBB bispecific antibodies enabled by innovative antibody engineering techniques have renewed hopes that these technologies may become available as broadly deployable platform to accelerate burgeoning biotherapeutic pipelines for CNS - the future for CNS antibody therapeutics has just become much brighter.

\section{Financial \& competing interests disclosure}

Biogen Idec and the National Research Council of Canada have a Sponsored Research Agreement to jointly develop FC5. NRC authors have no financial or managerial interest in the company. The authors have no other relevant affiliations or financial involvement with any organization or entity with a financial interest in or financial conflict with the subject matter or materials discussed in the manuscript apart from those disclosed.

No writing assistance was utilized in the production of this manuscript.

\section{References}

1 Paul SM, Mytelka DS, Dunwiddie CT et al. How to improve $R \& D$ productivity: the pharmaceutical industry's grand challenge. Nat. Rev. Drug Discov. 9, 203-214 (2010).
2 Arrowsmith J, Miller P. Trial watch: phase II and phase III attrition rates 2011-2012. Nat. Rev. Drug Discov. 12, 569 (2013).

3 Smith AJ. New horizons in therapeutic antibody discovery: opportunities and challenges versus small-molecule therapeutics. J. Biomol. Screen. doi:10.1177/1087057114562544 (2014) (Epub ahead of print).

4 Hampel H, Schneider LS, Giacobini E et al. Advances in the therapy of Alzheimer's 
disease: targeting amyloid beta and tau and perspectives for the future. Expert Rev. Neurother. 15, 83-105 (2015).

5 Tayeb HO, Murray ED, Price BH, Tarazi FI. Bapineuzumab and solanezumab for Alzheimer's disease: is the 'amyloid cascade hypothesis' still alive? Expert Opin. Biol. Ther. 13, 1075-1084 (2013).

6 Levites Y, Smithson LA, Price RW et al. Insights into the mechanisms of action of anti-Abeta antibodies in Alzheimer's isease mouse models. FASEB J. 20, 2576-2578 (2006).

7 Pardridge WM, Buciak JL, Friden PM. Selective transport of an anti-transferrin receptor antibody through the blood-brain barrier in vivo. J. Pharmacol. Exp. Ther. 259, 66-70 (1991)

8 Jones AR, Shusta EV. Blood-brain barrier transport of therapeutics via receptormediation. Pharm. Res. 24, 1759-1771 (2007).

9 Boado RJ, Lu JZ, Hui EK, Pardridge WM. Insulin receptor antibody-sulfamidase fusion protein penetrates the primate blood-brain barrier and reduces glycosoaminoglycans in
Sanfilippo type A cells. Mol. Pharm. 11, 2928-2934 (2014).

10 Yu YJ, Zhang Y, Kenrick M et al. Boosting brain uptake of a therapeutic antibody by reducing its affinity for a transcytosis target. Sci. Transl. Med. 3(84), 84 ra44 (2011).

11 Couch JA, Yu YJ, Zhang Y et al. Addressing safety liabilities of TfR bispecific antibodies that cross the blood-brain barrier. Sci. Transl. Med. 5, 183ra57, 1-12 (2013).

12 Yu YJ, Atwal JK, Zhang Y et al. Therapeutic bispecific antibodies cross the blood-brain barrier in nonhuman primates. Sci. Transl. Med. 6, 261ra154 (2014).

13 Niewoehner J, Bohrmann B, Collin L et al. Increased brain penetration and potency of a therapeutic antibody using a monovalent molecular shuttle. Neuron 81, 49-60 (2014).

14 ABBVIE, INC.: WO 2014089209 A2, AbbVie Deutschland GmbH \& Co. KG (2014).

15 Hasler J, McGowan E, Rutkowski L. Species cross-reactive single domain antibodies (VNARs) to the transferrin receptor 1 (TFR1) that cross the BBB. Presented at: Cold
Spring Harbor Blood-Brain Barrier Conference. Cold Spring Harbor, NY, USA, 10-13 December 2014 (Abstract book 32).

16 Tanha J, Muruganandam A, Stanimirovic D. Phage display technology for identifying specific antigens on brain endothelial cells. Methods Mol. Med. 89, 435-449 (2003).

17 Muruganandam A, Tanha J, Narang S, Stanimirovic D. Selection of phage-displayed llama single-domain antibodies that transmigrate across human blood-brain barrier endothelium. FASEB J. 16, 240-242 (2002).

18 Stanimirovic DB, Kemmerich K, Haqqani AS, Farrington GK. Engineering and pharmacology of blood-brain barrierpermeable bispecific antibodies. $A d v$. Pharmacol. 71, 301-335 (2014).

19 Farrington GK, Caram-Salas N, Haqqani AS et al. A novel platform for engineering blood-brain barrier-crossing bispecific biologics. FASEB J. 28, 4764-4778 (2014).

20 Iliff JJ, Wang M, Zeppenfeld DM et al. Cerebral arterial pulsation drives paravascular CSF-interstitial fluid exchange in the murine brain. J. Neurosci. 33, 18190-18199 (2013). 